\title{
Metacognition and Maladaptive Coping as Components of Test Anxiety
}

\author{
Gerald Matthews ${ }^{1 *}$, Emma J. Hillyard ${ }^{2}$ \\ and Sian E. Campbell ${ }^{2}$ \\ ${ }^{1}$ University of Cincinnati, Ohio, USA \\ ${ }^{2}$ University of Dundee, UK
}

\begin{abstract}
Text anxiety refers both to states of distress and worry during examinations, and to traits which predispose the person to state anxiety. Wells and Matthews (1994) have proposed a general model of emotional disorder which describes a variety of cognitive processes which may contribute to trait and state test anxiety. These processes include excessive metacognition, maladaptive coping and worry. A study was run to investigate relationships between cognitive processes and test anxiety. Eighty-four undergraduate students completed questionnaires relating to cognition and trait text anxiety prior to an examination. Their stress states and coping during the examination itself were also assessed. Results showed that trait test anxiety relates to two distinct cognitive factors, relating to (i) metacognition and worry, and (ii) maladaptive coping. Cognitive measures also predicted some aspects of state response to the examination setting. Relationships between cognition and test anxiety were generally consistent with the model of Wells and Matthews (1994). Results also suggested that treatment for pathological test anxiety should reflect its cognitive basis. For example, test anxiety based on metacognitive dysfunction may require use of cognitivebehavioural techniques used to treat more general anxiety conditions. Conversely, test anxiety derived from maladaptive coping may respond best to training in coping skills. Attention training techniques may contribute to therapy for both metacognition and coping-based disorder. Copyright (C) 1999 John Wiley \& Sons, Ltd.
\end{abstract}

\section{INTRODUCTION}

Test anxiety is normally defined in terms of its subjective symptoms, i.e. the person's awareness of physiological, emotional and cognitive functioning (see Zeidner, 1998, for a review). There appear to be distinct, though correlated, symptom dimensions at both trait and state levels. The predisposition to test

*Correspondence to: Gerald Matthews, Department of Psychology, University of Cincinnati, Ohio 45221-0376, USA. Email address: matthegd@email.uc.edu

Contract grant sponsor: Medical Research Council. Contract grant number: G9510930. anxiety, i.e. the trait, is often assessed with Sarason's (1984) Reaction to Tests (RTT) questionnaire, which distinguishes Bodily Reactions, such as a racing heart, Tension (negative emotion) and two cognitive dimensions, Worry and Test-Irrelevant Thinking. Worry refers to concerns about failure, and Test-Irrelevant Thinking to thoughts about matters unrelated to the test. The cognitive components of test anxiety are considered the most damaging to performance, and provide the focus for clinical interventions which seek to re-direct attention from distracting thoughts to the task at hand (e.g. Sarason et al., 1990). Cognitively 
informed assessment of traits such as test anxiety helps the clinician to understand the person, to select therapies compatible with the patient's dispositions and to anticipate the course of therapy (Matthews et al., 1998).

Test anxiety is also experienced as short-lived states associated with a specific examination situation. Sarason's work has focused on its cognitive expression. The Cognitive Interference Questionnaire (CIQ: Sarason et al., 1986) assesses task-related and task-irrelevant thoughts. Emotional or moodrelated reactions may include tension, unhappiness and loss of energy. Test anxiety may also have a motivational aspect. Although anxiety is sometimes motivating, test anxiety seems to be typically accompanied by loss of interest in the task and desire to withdraw from the situation (Geen, 1987).

The theory of test anxiety emphasizes selfpreoccupation, especially with personal inadequacies, and self-focused attention (Sarason et al., 1990). However, Sarason's empirical studies have investigated test anxiety mainly at the level of the multiple symptoms discriminated by the RTT. It is unclear how far the self-preoccupation syndrome described by Sarason et al. (1990) might be decomposed into distinct constituent processes. Other authors have linked test anxiety to various selfrelated constructs such as self-esteem, self-efficacy and self-concept (e.g. Glass et al., 1995), and expectancy and goal engagement (Carver and Scheier, 1992). Cognitive interference may also relate to appraisal and coping processes (Wells and Matthews, 1994). Metacognition may be of special relevance to test anxiety, given that (i) worry is central to test anxiety (Sarason et al., 1986) and (ii) one of the salient features of clinical anxiety conditions is beliefs concerning diminished cognitive control (Wells, 1997). In clinical conditions such as Generalized Anxiety Disorder (GAD) selfpreoccupation seems to result from metacognitive beliefs about the utility of worry and self-evaluation (Wells, 1994). Discrimination of these various possible components of test anxiety would allow more effective targeting of clinical interventions.

\section{Discriminating Components of Test Anxiety within the S-REF Model}

It is important to determine whether test anxiety is controlled by a single key cognitive process, or whether multiple, functionally distinct processes make independent contributions to anxiety. Test anxiety theory should also differentiate the predisposition to become test anxious, i.e. trait test anxiety, from the immediate state of being test anxious in a particular situation. Next, we discuss a theoretical framework which supports these distinctions. The Self-Referent Executive Function (S-REF) model of emotional disorder developed by Wells and Matthews (1994, 1996) describes a cognitive architecture for information processing in states of distress and worry. This multi-level architecture may provide a useful framework for systematizing the diverse cognitive aspects of test anxiety, including metacognition, and guiding therapy. Figure 1 shows the model, which comprises three interacting levels. Low-level or 'automatic' processing networks generate intruding thoughts, which activate the executive system (S-REF), which constitutes the second level. The S-REF initiates and controls self-regulative processes including appraisal of personal significance and implications of events, and choice of coping strategy. It also performs the metacognitive functions of monitoring internal state, and attempting to intensify or suppress significant thoughts. S-REF functioning is critically dependent upon the third level, self-beliefs in long-term memory (LTM), roughly corresponding to the 'self-schema'. Beliefs may be declarative in form, or they may be encoded within 'plans' or generic self-regulative procedures. The S-REF accesses situationally-relevant plans and adapts them to prevailing circumstances, an attentionally demanding process which may distract from other activities. Evaluative situations may trigger plans for scrutiny of thoughts and emotions in test anxious individuals, leading to maladaptive coping that withdraws attention from the task at hand.

The S-REF architecture supports a variety of distinct processes, including metacognition, appraisal, regulation of coping and accessing selfbeliefs, which, in turn, are influenced by the plans comprising self-knowledge. Hence, the model may permit us to distinguish different processing functions contributing to test anxiety. The S-REF model incorporates the distinction of Nelson and Narens (1994) between monitoring and control within a metacognitive system. Monitoring refers to the flow of information from processing to a 'meta-level' executive, i.e. the S-REF itself in the WellsMatthews (1994) model. Measures of metacognition used in clinical contexts (e.g. Cartwright-Hatton and Wells, 1997) seem to refer primarily to the typical outputs of the monitoring process, such as beliefs that thoughts are themselves a source of threat. The S-REF model emphasizes that metacognitive beliefs are not just a passive registration of inputs from lower-level processing. Beliefs are 


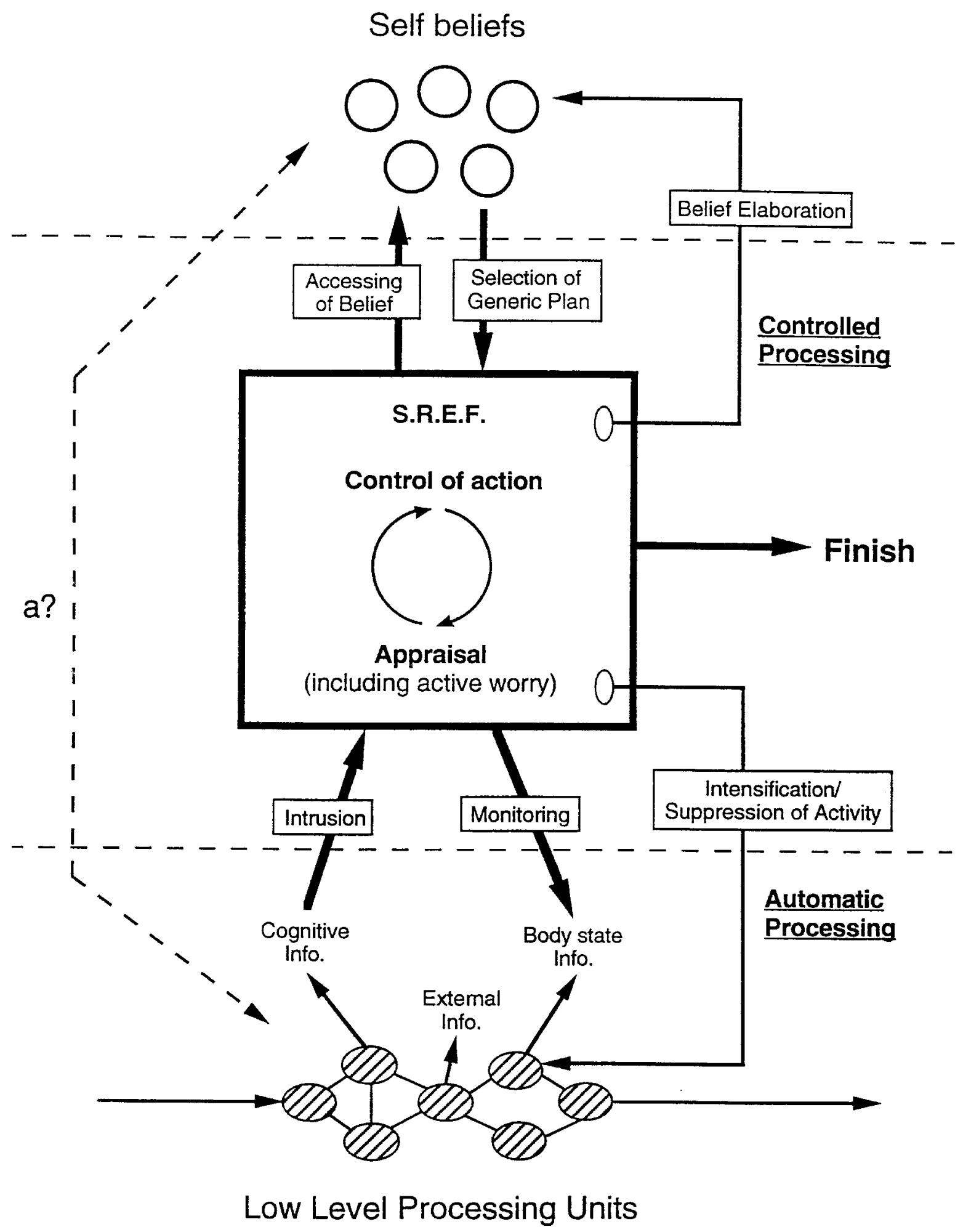

Figure 1. The Self-Regulatory Executive Function (S-REF) Model of emotional disorder. (Reproduced from Wells and Matthews, 1994, p. 268.) 
actively constructed by plans retrieved from selfknowledge.

Control refers to the executive function of strategic modification of lower-level processing, on the basis of the information received through monitoring (Nelson and Narens, 1994). In the S-REF model, strategic intervention is implemented via coping. There are various taxonomies of coping, but there is some agreement in distinguishing task-focus (or problem-focus), emotion-focus and avoidance as basic dimensions (Endler and Parker, 1990). Taskfocus relates to changing the external environment through systematic action, emotion-focus to altering one's feelings and thoughts about events and avoidance to suppressing or distracting oneself from awareness of the problem. Task-focus is likely to be more adaptive than the other two strategies in coping with test anxiety, because this strategy directs attention towards the task at hand, whereas emotion-focus and avoidance withdraw attention from the task.

The third construct of interest, worry, may be conceptualized as a broad syndrome of processing relating to both monitoring and control. Dynamically, metacognition/monitoring may be seen as feeding into coping/control, which then feeds back into metacognition. Choosing to cope through strategies such as rumination and thought suppression is likely to enhance metacognitive awareness (Wells and Matthews, 1994). Worry describes an overall configuration of this cycle, related to both monitoring and coping. Specifically, worry relates to (i) awareness of intrusive, predominantly negative ideation of varying content, (ii) metacognitive beliefs relating to the personal significance of intrusive or negative thoughts and (iii) coping through perseverative rumination, as a form of problem-solving (Matthews and Wells, 1996). Wells (1994, 1997) identifies metaworry (worry about one's own worry) as an aspect of worry that may be especially implicated in anxiety disorders such as GAD.

In summary, we expect metacognition and coping to overlap because the content of metacognitive beliefs will influence choice of coping strategy (and choice of coping may enhance or reduce metacognitive awareness). Worry should overlap with (i) metacognition and (ii) coping because worry is a superordinate construct associated with both metacognition and coping.

\section{Trait and State Aspects of Test Anxiety}

The essence of the trait conception of test anxiety is that some individuals are consistently vulnerable to anxiety across a variety of evaluative situations (Sarason, 1984). Such individuals may be prone to react to such situations with the maladaptive metacognitive and coping processes described by the S-REF model, so there is a further issue concerning the sources of this vulnerability to maladaptive processing within test settings. Matthews et al. (in press-b) describe a cognitive theory of personality traits, based on the S-REF model, which proposes that personality reflects consistencies of self-knowledge, i.e. stable biases in the plans which provide the basis for situational processing. Typically, traits relate to multiple components of self-knowledge. For example, neuroticism seems to relate to negative declarative self-beliefs, and to plans for appraising events as threatening and for coping through self-criticism. Activation of these plans, and hence the pattern of cognitive bias shown by more neurotic subjects, depends on situational factors, such as a threat to self-esteem. Similarly, there may be various sources of test anxiety, including negative beliefs about personal competence in the test environment, and plans for dysfunctional coping. These beliefs and plans will be prone to activation in evaluative environments, leading to test anxiety in vulnerable individuals.

At the state level, it is important to distinguish underlying processes, such as those implementing monitoring and control, from the person's immediate experience of mental disturbance. Disturbed states are often characterized in terms of rather vague constructs such as 'stress' and 'anxiety'. However, such constructs are often too broad and ill defined to be very useful. For example, 'state anxiety' covers both tension and worry (Sarason, 1984). It may also refer both to states in which the person is motivated to compensate through taskdirected effort, and states in which the primary motivation is to withdraw from the situation, with very different behavioural consequences (e.g. Geen, 1987). Recent work (Matthews et al., in press-a) suggests that anxiety and stress states may be described in terms of three broad syndromes of task (dis)engagement, distress and state worry, defined by dimension related to the traditional domains of emotion (mood), motivation and cognition. In this context, 'state worry' refers to the person's experience of stress/anxiety symptoms related to intruding thoughts, rather than the metacognitive and coping processes believed to underlie awareness of thoughts. The cognitive interference construct of Sarason et al. (1986) is central to state worry. The three state syndromes appear to reflect distinct 
Table 1. Three stress state syndromes, and their associations with appraisal and coping measures (Matthews et al., in press-a).

\begin{tabular}{llll}
\hline & Task engagement & Distress & Worry \\
\hline Principal scales & $\begin{array}{l}\text { Energetic arousal } \\
\text { Motivation } \\
\text { Concentration }\end{array}$ & $\begin{array}{l}\text { Tense arousal } \\
\text { Low hedonic tone }\end{array}$ & $\begin{array}{l}\text { Self-focus of attention } \\
\text { Low self-esteem }\end{array}$ \\
& Low confidence and control & $\begin{array}{l}\text { CI (task-related) } \\
\text { CI (task-irrelevant) }\end{array}$ \\
Appraisals & High cognitive demands & High overall workload & Self-referent appraisals \\
& High effort & Threat & \\
Coping & Task-focus & Emotion-focus & Emotion-focus \\
& & & Avoidance \\
\hline
\end{tabular}

Note. CI, Cognitive Interference.

styles of appraisal and coping in performance settings. Table 1 summarizes the nature of the three stress state syndromes, on the basis of a series of empirical studies (Matthews et al., in press). Use of this taxonomy of states may indicate how test anxiety is actually experienced in a given setting, or within a given individual.

\section{Overview and Aims}

The principal aim of the study was to test whether metacognition relates to test anxiety in a student sample. We also aimed to test whether test anxiety relates to multiple cognitive components, consistent with the framework of Matthews et al. (in press-b) for personality theory. The study compared metacognition with coping and worry measures as predictors of test anxiety. There were two phases of testing. First, student subjects completed Sarason's (1984) RTT and various cognitive measures several weeks before an examination. The cognitive measures comprised multidimensional questionnaires for metacognition, assessed with the Meta-Cognitions Questionnaire (MCQ: Cartwright-Hatton and Wells, 1997), coping, assessed with the dispositional Coping Inventory for Task Stress (CITS-D: Matthews and Campbell, 1998) and trait worry, assessed with the Anxious Thoughts Inventory (AnTI: Wells, 1994). Subsequently, we will use 'cognitive traits' as a shorthand term for the MCQ, AnTI and CITS-D scales. Strictly, the CITS-D assesses stable dispositions within a particular context (demanding tasks) rather than a trait generalizing across many situations.

In the second phase, subjects completed a multidimensional stress state measure immediately after the examination, using the Dundee Stress State Questionnaire (DSSQ: Matthews et al., 1996, in press-a), which measures the stress state components listed in Table 1. We also assessed subjects' cognitions of the examination-their appraisals of the workload imposed by the task (NASA-TLX: Hart and Staveland, 1988) and their coping with the specific test situation, assessed with the situational Coping Inventory for Task Stress (CITS-S: Matthews and Campbell, 1998).

The study was predominantly exploratory, but some tentative predictions were framed using the general account of anxiety provided by the S-REF model. Predictions were directed towards (i) bivariate relationships between trait test anxiety and other trait-like cognitive dimensions, (ii) multivariate relationships between test anxiety and cognition and (iii) individual differences in state response during the examination.

\section{Trait test anxiety and 'cognitive traits'}

Metacognition, worry and maladaptive coping are central to anxiety disorders. Test anxious individuals, i.e. high scorers on the RTT, should be generally more inclined to see metacognitions as salient and personally significant, and so should obtain higher MCQ scores. Similarly, AnTI scales should relate positively to test anxiety, because of the key role of worry in anxiety. (Note that RTT Worry is conceptually distinct from the AnTI scales in that it measures predisposition to awareness of thoughts specifically in evaluative settings.) The AnTI includes a metaworry scale (worry about worry), which was expected to be especially predictive, because thoughts about control of intrusions are likely to be salient in test settings. Test anxiety should also relate to a maladaptive style of 
coping, i.e. use of emotion-focus and avoidance, at the expense of task-focus.

Multivariate analysis of test anxiety and cognition

The study aimed to test formally the prediction that test anxiety should be independently related to multiple aspects of metacognition, coping and worry. Multivariate testing involved three stages. First, we factor analysed the MCQ, AnTI and CITS-D to simplify the cognitive data. Second, we tested whether test anxiety was related to single or multiple factors, using multiple regression. Third, we tested whether residual variance in the MCQ, AnTI and CITS-D predicted additional variance in test anxiety, over and above the variance predicted by the factors.

\section{Individual differences in state response}

Previous test anxiety research (see Sarason et al., 1990) suggests that, in terms of the DSSQ constructs, states of test anxiety are expressed most strongly as distress (e.g. tension) and state worry (e.g. cognitive interference). It was expected that the MCQ and AnTI should predict the DSSQ state worry scales most strongly. The MCQ and AnTI should predict maladaptive coping during the examination, because worry tends to be associated with use of emotion-focus and avoidance, at the expense of task-focus (Wells and Matthews, 1994). Metacognition and worry should relate also to higher appraised workload, because uncontrolled intrusive thoughts are likely to increase perceptions of task difficulty.

\section{METHOD}

\section{Subjects}

Subjects were 84 University of Dundee undergraduates, 28 men and 56 women, studying chemistry, life science or biochemistry courses. Mean age was 20.0 years (range, $17-48$ ).

\section{Materials}

The following trait questionnaires were used.

1. MCQ (Cartwright-Hatton and Wells, 1997). The MCQ comprises 65 items related to metacognition. It assesses (i) Positive Beliefs (that worry is helpful), (ii) Uncontrollability and Danger (negative beliefs about controllability and dangers of thoughts), (iii) Cognitive Confidence (high scores indicate beliefs that cognitive failure is likely), (iv) Superstition, Punishment and Responsibility (SPR: negative beliefs relating to these themes) and (v) Cognitive SelfConsciousness. The MCQ has been validated in both normal and clinical groups. Scale reliabilities (alpha coefficients) range from 0.72 to 0.89 .

2. AnTI (Wells, 1994). The 22-item AnTI assesses two content aspects of worry, Social and Health worries, and a more process-oriented dimension of Meta-Worry, i.e. worry about worry. These scales correlate with other measures related to anxiety in nonpatients, and discriminate between different patient groups. Reliabilities range from 0.75 to 0.84 . Cartwright-Hatton and Wells (1997) confirmed that MCQ and AnTI are positively correlated.

3. CITS-D (Matthews and Campbell, 1998). The Coping Inventory for Task Situations (CITS) was developed for assessment of the three Endler-Parker (1990) dimensions in performance related settings, on the principle that coping measures should relate to some specific situation or context of interest (Costa et al., 1996). There are both dispositional (CITS-D) and situational (CITS-S) versions, each with 21 items. Scale development of the 21-item CITS has focused mainly on the situational measure. However, in a recent study $(N=48)$, we administered the CITS with dispositional instructions and found that the Task-Focus, Emotion-Focus and Avoidance scales remained highly reliable (range, 0.86-0.88), and appropriately correlated with personality measures. Subjects were asked to rate their typical use of coping strategies in dealing with difficulties arising from tests and examinations.

4. RTT (Sarason, 1984). The RTT is made up of 40 items and assesses the person's usual reactions to tests and examinations. Its scales are Tension, Worry, Test-Irrelevant Thinking and Bodily Symptoms (range of reliabilities, 0.68-0.81). Relationships between the RTT and cognitive interference and task performance appear to vary with instructional manipulations as predicted by test anxiety theory.

Reactions to the examination were assessed with the following.

1. DSSQ (Matthews et al., in press-a). The DSSQ aims to provide comprehensive assessment of 
affective, motivational and cognitive states in performance settings. It has ten factorial scales. Second-order factor analysis shows that the scales may be grouped into the three broad stress state syndromes of task (dis)engagement, distress and worry. Scale reliabilities range from 0.76 to 0.89 . The two Cognitive Interference scales are shortened versions (eight items each) of the slightly longer scales developed by Sarason et al. (1986). The present study used a modified version of the Matthews et al. (in press-a) Motivation scale, which assessed intrinsic motivation (e.g. interest in the task performed). The DSSQ scales have been validated in experimental studies of task-induced stress. They also correlate appropriately with a variety of other individual difference measures, including scales for personality, appraisal and coping.

2. CITS-S (Matthews and Campbell, 1998). The CITS-S comprises 21 items assessing Task-Focus (e.g. applying systematic effort to the task), Emotion-Focus (e.g. blaming oneself for difficulties) and Avoidance (e.g. not taking the task too seriously). Instructions require the subject to rate how they coped with a task they have just completed (i.e. a single-task situation). Matthews and Campbell (1998) showed the scales had a strong factorial basis, in a sample of 456 subjects performing various laboratory tasks. Reliabilities vary between 0.84 and 0.86 . The scales are appropriately sensitive to both task and personality factors.
3. NASA-TLX (Hart and Staveland, 1988). The NASA-TLX is a standard workload measure. It was used in this study to assess individual differences in appraisal of the cognitive demands of the examination. The respondent rates six aspects of task load, which are combined to give an overall score. This study used a modified 0-10 rating scale for response, and calculated overall workload as an unweighted mean of the ratings (see Hendy et al., 1993, for a discussion of psychometric properties of the NASA-TLX).

\section{Design and Procedure}

Subjects completed questionnaires on two occasions. On the first occasion, two to three weeks prior to the examination, the MCQ, AnTI, CITS-D and RTT were completed. On the second occasion, the DSSQ and CITS-S were completed immediately after a University examination, at the same desk as used for the examination. Depending on the course taken, the result of the examination influenced either acceptance onto future courses or final degree examination grade.

\section{RESULTS}

\section{Intercorrelations of Cognitive Trait Measures}

Table 2 gives the intercorrelations of MCQ, AnTI and CITS-D 'cognitive trait' scales. The MCQ and

Table 2. Intercorrelations of MCQ, AnTI and CITS-D scales.

\begin{tabular}{|c|c|c|c|c|c|c|c|c|c|c|c|}
\hline & 1 & 2 & 3 & 4 & 5 & 6 & 7 & 8 & 9 & 10 & 11 \\
\hline 1. Positive Beliefs & 一 & & & & & & & & & & \\
\hline $\begin{array}{l}\text { 2. Uncontrollability } \\
\text { and Danger }\end{array}$ & $0.28 * *$ & - & & & & & & & & & \\
\hline $\begin{array}{l}\text { 3. Cognitive } \\
\text { Confidence }\end{array}$ & 0.18 & $0.43 * *$ & 一 & & & & & & & & \\
\hline 4. $\mathrm{SPR}^{\mathrm{a}}$ & $0.38 * *$ & $0.52 * *$ & $0.23 *$ & - & & & & & & & \\
\hline $\begin{array}{l}\text { 5. Cognitive Self- } \\
\text { Consciousness }\end{array}$ & $0.33^{* *}$ & $0.39 * *$ & 0.09 & $0.41 * *$ & - & & & & & & \\
\hline 6. Social Worry & $0.34 * *$ & $0.55^{* *}$ & $0.37 * *$ & $0.26^{*}$ & $0.33 * *$ & - & & & & & \\
\hline 7. Health Worry & $0.30 * *$ & $0.38 * *$ & $0.24 *$ & $0.30 * *$ & $0.26^{*}$ & $0.42 * *$ & - & & & & \\
\hline 8. Meta-Worry & $0.32 * *$ & $0.71 * *$ & $0.39 * *$ & $0.38 * *$ & $0.37 * *$ & $0.59 * *$ & $0.56^{* *}$ & - & & & \\
\hline 9. Task-Focus & 0.11 & -0.06 & $-0.37 * *$ & 0.13 & $0.30 * *$ & -0.18 & -0.02 & -0.12 & - & & \\
\hline 10. Emotion-Focus & $0.25^{*}$ & $0.52 * *$ & $0.40 * *$ & $0.28 * *$ & 0.14 & $0.61 * *$ & $0.42 * *$ & $0.53 * *$ & $-0.32 * *$ & - & \\
\hline 11. Avoidance & -0.08 & 0.08 & 0.12 & 0.03 & -0.10 & 0.05 & -0.02 & 0.06 & $-0.23^{*}$ & $0.24^{*}$ & - \\
\hline
\end{tabular}

Note. $* p<0.05, * * p<0.01$.

${ }^{a}$ Negative beliefs about thoughts in general, including themes of superstition, punishment and responsibility. 
AnTI were substantially intercorrelated. Of the CITS-D coping scales, Task-Focus correlated positively with Cognitive Self-Consciousness, and negatively with Cognitive Confidence (i.e. Task-Focus relates to high confidence). Emotion-Focus was significantly positively correlated with all the MCQ and AnTI scales except Cognitive SelfConsciousness. It related most strongly to the AnTI scales and to Uncontrollability and Danger. Avoidance was unrelated to the MCQ and AnTI scales.

In view of the correlations between the cognitive trait scales, a factor analysis was conducted. Horn's (1966) parallel analysis, a method validated against artificial data by Zwick and Velicer (1986), showed that two factors should be extracted from the interscale correlation matrix shown in Table 2. An initial principal component solution was rotated using the direct oblimin method, which permits oblique factors. The factor pattern matrix is shown in Table 3, together with variable communalities. The two factors explained $53.8 \%$ of the variance, and were largely independent (correlation $=-0.05$ ). The first factor appeared to be a general Metacognition factor, with substantial loadings for all MCQ and AnTI scales, and for CITS-D Emotion-Focus. Highest loadings were for MCQ Uncontrollability and Danger and AnTI Meta-Worry. The second factor was labelled 'Adaptive Coping'. It contrasted use of Task-Focus with use of Emotion-Focus and Avoidance. It also had two loadings greater than 0.4 for MCQ scales: low Cognitive Confidence and high Cognitive Self-Consciousness. The pattern of maladaptive coping defined by the CITS-D variables also related to negative beliefs about cognitive efficiency, and to low self-focused attention. Factor scores were calculated for each subject for use in further analyses, using the standard regression method.

\section{Cognitive Trait Predictors of the RTT}

RTT test anxiety scales were related to all three of the cognitive trait questionnaires (see Table 4). All four scales were correlated with Uncontrollability and Danger, Cognitive Confidence, Meta-Worry and Emotion-Focus. Other cognitive scales were more selectively related to the RTT. For example, Positive Beliefs was significantly correlated with the less cognitive aspects of test anxiety (Tension and Test-Irrelevant Thinking), whereas Task-Focus was negatively associated with its explicitly cognitive dimensions (Worry and Test-Irrelevant Thinking). Avoidance was significantly correlated only with Test-Irrelevant Thinking. At the level of factor scores, Metacognition predicted all RTT scales, although the correlation with Test-Irrelevant Thinking was small in magnitude. Adaptive Coping was associated with lower Worry and lower TestIrrelevant Thinking.

\section{Multivariate Analysis of Test Anxiety and Cognitive Traits}

So far, it has been shown that test anxiety overlaps with metacognition, worry and coping. The next stage of the analysis was to discriminate independent predictors of test anxiety, using multiple regressions. The regression analysis had two aims. The first aim was to test whether the cognitive trait factor scores were independently related to the RTT scales. The second aim was to test whether overlap between test anxiety and the cognitive trait scales could be fully explained by the factor scores, or,

Table 3. Factor structure of MCQ, AnTI and CITS-D scales (factor pattern matrix).

\begin{tabular}{lccc}
\hline & Factor 1 (Metacognition) & Factor 2 (Adaptive Coping) & Communality \\
\hline Positive Beliefs & 0.57 & 0.27 & 0.39 \\
Uncontrollability and Danger & 0.81 & -0.10 & 0.67 \\
Cognitive Confidence & 0.47 & -0.48 & 0.47 \\
SPR & 0.65 & 0.25 & 0.47 \\
Cognitive Self-Consciousness & 0.61 & 0.50 & 0.58 \\
Social Worry & 0.73 & -0.22 & 0.60 \\
Health Worry & 0.65 & -0.02 & 0.42 \\
Meta-Worry & 0.81 & -0.14 & 0.70 \\
Task-Focus & 0.01 & 0.83 & 0.68 \\
Emotion-Focus & 0.64 & -0.47 & 0.66 \\
Avoidance & 0.00 & -0.52 & 0.27 \\
\hline
\end{tabular}

Note. ${ }^{2}$ Negative beliefs about thoughts in general, including themes of superstition, punishment and responsibility. 
Table 4. Correlations of MCQ, AnTI and CITS-D scales and factor scores with RTT scales.

\begin{tabular}{|c|c|c|c|c|}
\hline & \multicolumn{4}{|c|}{ RTT Scales } \\
\hline & Tension & Worry & $\begin{array}{l}\text { Test-Irrelevant } \\
\text { Thinking }\end{array}$ & $\begin{array}{c}\text { Bodily } \\
\text { Symptoms }\end{array}$ \\
\hline \multicolumn{5}{|l|}{ Scales } \\
\hline Positive Beliefs & $0.38 * *$ & 0.14 & -0.01 & $0.35 * *$ \\
\hline Uncontrollability and Danger & $0.49 * *$ & $0.52 * *$ & $0.32 * *$ & $0.57 * *$ \\
\hline Cognitive Confidence & $0.32 * *$ & $0.48 * *$ & $0.41 * *$ & $0.31 * *$ \\
\hline $\mathrm{SPR}^{\mathrm{a}}$ & $0.34 * *$ & $0.26^{* *}$ & -0.01 & $0.37 * *$ \\
\hline Cognitive Self-Consciousness & $0.24^{*}$ & $0.24^{*}$ & -0.02 & $0.43^{* *}$ \\
\hline Social Worry & $0.48^{* *}$ & $0.42 * *$ & 0.17 & $0.44 * *$ \\
\hline Health Worry & $0.26^{*}$ & $0.23^{*}$ & 0.16 & $0.23^{*}$ \\
\hline Meta-Worry & $0.53 * *$ & $0.51 * *$ & $0.23 *$ & $0.53 * *$ \\
\hline Task-Focus & -0.04 & $-0.31 * *$ & $-0.46^{* *}$ & 0.07 \\
\hline Emotion-Focus & $0.60 * *$ & $0.59 * *$ & $0.29 * *$ & $0.45^{* *}$ \\
\hline Avoidance & -0.09 & 0.03 & $0.27 *$ & 0.03 \\
\hline \multicolumn{5}{|l|}{ Factor scores } \\
\hline Metacognition & $0.61 * *$ & $0.55^{* *}$ & $0.22 *$ & $0.62 * *$ \\
\hline Adaptive Coping & -0.11 & $-0.34 * *$ & $-0.49 * *$ & 0.01 \\
\hline
\end{tabular}

Note. ${ }^{*} p<0.05, * * p<0.01$.

${ }^{a}$ Negative beliefs about thoughts in general, including themes of superstition, punishment and responsibility.

alternatively, whether the individual scales predicted additional variance. To prevent collinearity between the factor scores and scales, each scale was residualized against the two factors prior to analysis. The smaller the communality of the scale (see Table 3), the greater the proportion of scale variance associated with the residual, rather than with the common factors. Separate equations were constructed for each of the four RTT scales, using the following method. First, both factor scores were entered. Second, we tested whether each of the three sets of residuals (MCQ, AnTI and CITS-D) added significantly to the variance explained. Any set of residuals that did so was included in the final regression equation.

Summary statistics are given in Table 5 . In each case, $R$ was significant, with the equations explaining from 28 to $48 \%$ of the variance in the test anxiety dimensions. The lower part of the Table indicates which predictors were significant in the

Table 5. Stepwise regressions of RTT scales onto factor scores and residualized CITS-D scales: summary statistics for final equations (upper part) and $\beta$ weights for individual predictors (lower part).

\begin{tabular}{|c|c|c|c|c|}
\hline & \multicolumn{4}{|c|}{ Criterion RTT scale } \\
\hline & Tension & Worry & $\begin{array}{l}\text { Test-Irrelevant } \\
\text { Thinking }\end{array}$ & $\begin{array}{c}\text { Bodily } \\
\text { Symptoms }\end{array}$ \\
\hline \multicolumn{5}{|l|}{ Summary statistics } \\
\hline$R$ & 0.69 & 0.63 & 0.53 & 0.62 \\
\hline$R^{2}$ & 0.48 & 0.39 & 0.28 & 0.39 \\
\hline$F$ & $14.3 * *$ & $26.4^{* *}$ & $15.8^{* *}$ & $25.3^{* *}$ \\
\hline$d f$ & 5,78 & 2,81 & 2,81 & 2,81 \\
\hline \multicolumn{5}{|l|}{$\beta$ weights } \\
\hline Metacognition (Factor 1) & $0.60 * *$ & $0.53^{* *}$ & $0.20 *$ & $0.62 * *$ \\
\hline Adaptive Coping (Factor 2) & -0.07 & $0.31 * *$ & $-0.48 * *$ & 0.01 \\
\hline Task-Focus (Residual) & 0.06 & - & - & - \\
\hline Emotion-Focus (Residual) & $0.25^{* *}$ & - & - & - \\
\hline Avoidance (Residual) & $-0.19^{*}$ & - & - & - \\
\hline
\end{tabular}

Note. $* p<0.05, * * p<0.01$.

$\beta$ weights for predictors refer to the final equations. 
Table 6. Correlations of MCQ, AnTI and CITS-D scales and factor scores with DSSQ scales.

\begin{tabular}{|c|c|c|c|c|c|c|c|c|c|c|}
\hline & Tension & HT & Confidence & Energy & Motivation & Concen. & Self-focus & Esteem & CI-TR & CI-TI \\
\hline \multicolumn{11}{|l|}{ Scales } \\
\hline Positive Beliefs & 0.05 & 0.07 & 0.06 & 0.02 & 0.05 & -0.02 & $0.22 *$ & -0.10 & 0.04 & 0.18 \\
\hline Uncontrollability and & 0.15 & -0.08 & -0.16 & -0.15 & -0.20 & -0.14 & 0.08 & -0.06 & 0.20 & 0.17 \\
\hline \multicolumn{11}{|l|}{ Danger } \\
\hline Cognitive Confidence & $0.32 * *$ & -0.09 & -0.03 & -0.05 & 0.00 & -0.17 & 0.05 & -0.14 & 0.04 & 0.03 \\
\hline $\mathrm{SPR}^{\mathrm{a}}$ & -0.03 & 0.00 & -0.13 & -0.01 & $-0.25^{*}$ & -0.05 & $0.23 *$ & -0.04 & 0.16 & 0.16 \\
\hline Cognitive Self- & -0.11 & 0.13 & 0.00 & 0.05 & -0.16 & 0.02 & $0.22 *$ & -0.11 & 0.17 & 0.20 \\
\hline \multicolumn{11}{|l|}{ Consciousness } \\
\hline Social Worry & 0.12 & -0.07 & -0.06 & -0.14 & -0.18 & 0.02 & 0.06 & -0.07 & 0.21 & 0.13 \\
\hline Health Worry & 0.01 & -0.05 & -0.03 & -0.09 & -0.04 & 0.04 & $0.23^{*}$ & -0.04 & $0.22 *$ & 0.20 \\
\hline Meta-Worry & $0.22 *$ & -0.10 & -0.08 & -0.13 & -0.16 & -0.06 & 0.07 & -0.04 & $0.32 * *$ & 0.14 \\
\hline Task-Focus & $-0.37 * *$ & $0.32 * *$ & $0.28 * *$ & $0.22 *$ & 0.06 & $0.24 *$ & 0.01 & $0.28^{* *}$ & -0.04 & -0.07 \\
\hline Emotion-Focus & $0.29 * *$ & -0.20 & $-0.28 * *$ & $-0.26^{*}$ & -0.13 & -0.14 & 0.03 & -0.14 & $0.25^{*}$ & 0.07 \\
\hline Avoidance & $0.30 * *$ & $-0.24 *$ & -0.20 & -0.20 & -0.05 & -0.04 & 0.00 & -0.03 & 0.06 & 0.11 \\
\hline \multicolumn{11}{|l|}{ Factor scores } \\
\hline Metacognition & 0.14 & -0.05 & -0.11 & -0.12 & -0.19 & -0.06 & 0.19 & -0.10 & $0.28^{*}$ & $0.22 *$ \\
\hline Adaptive Coping & $-0.45^{* *}$ & $0.34 * *$ & $0.27 *$ & $0.26^{*}$ & 0.02 & 0.16 & 0.11 & 0.16 & -0.06 & 0.01 \\
\hline
\end{tabular}

Note. ${ }^{*} p<0.05, * * p<0.01$.

HT, Hedonic Tone; Concen., Concentration; CI, Cognitive Interference; TR, Task-Related; TI, Task-Irrelevant.

aNegative beliefs about thoughts in general, including themes of superstition, punishment and responsibility.

final regression equations. Contributions of the factors paralleled the correlations shown in Table 4, i.e. Worry and Test-Irrelevant Thinking related independently to Metacognition and the low Adaptive Coping. For Tension, entering the CITS-D residuals added significantly to the variance explained: $R^{2}$ increased by $11 \%(F(3,78)=5.22$, $p<0.01$ ) from 37 to $48 \%$. With inclusion of the residuals, the contribution of the Metacognition factor remained significant. In addition, high Emotion-Focus and low Avoidance were independently predictive of high RTT Tension. These analyses confirm that test anxiety is related to several distinct aspects of metacognition and cognition.

\section{State Response to the Examination}

The first step in analysing the data from the examination was to assess how subjective stress and anxiety were experienced. Matthews et al. (in press-a) collected 'normative' data for large sample $(N=517)$ tested on the DSSQ before performing various laboratory tasks, which provide a baseline for assessment of situational stress. Twosample $z$-tests showed significant $(p<0.01)$ deviations from baseline for the following scales (means for examination given first). Higher scores were obtained following the examination for tension (means, 21.6 vs 16.6; difference, 1.06 normative SD units) and for task-related cognitive interference (means, 21.9 vs 19.2; 0.44 SD). Lower scores were obtained following the examination for motivation (means, 14.6 vs $18.1 ;-0.69$ SD), hedonic tone (means, 19.8 vs $24.4 ;-1$ SD), confidence and control (means, 11.0 vs $13.4,-0.52$ SD) and selffocus (means, 6.9 vs 10.8; $-0.58 \mathrm{SD}$ ). In other words, subjects experienced negative moods and cognitions, mainly associated with the distress syndrome assessed by DSSQ, as well as low motivation (in the sense of low interest in the task). However, self-focus of attention was depressed, implying that some aspects of the stress state response were adaptive.

\section{Predictors of State Test Anxiety}

Several correlational analyses of the postexamination measures were performed. Correlations between the RTT and DSSQ scales (i.e. trait vs state test anxiety) were modest. RTT Worry was the only trait scale substantially predictive of the DSSQ. It was significantly correlated with all the scales except Self-Consciousness and Task-Irrelevant Interference, with correlation magnitudes ranging from 0.32 (Task-Related Interference; $p<0.01$ ) to -0.29 (Concentration; $p<0.01$ ). The only significant correlates of the remaining RTT scales was Motivation, which correlated negatively with Bodily Symptoms $(r=-0.25 ; p .<0.05)$, and TaskRelated Interference, which correlated with Tension $(r=0.24 ; p<0.05)$. We also checked whether the RTT related to appraisal of workload (NASA-TLX) and coping with the examination (CITS-S). RTT Worry was significantly correlated with higher workload $(r=0.28 ; p<0.05)$ and with emotion- 
focused coping $(r=0.28 ; p<0.05)$, but other RTT scales were not predictive.

Table 6 shows how the MCQ, AnTI and CITS-D scales related to the DSSQ. Significant correlations were of modest magnitude. The CITS-D was the best predictor of the distress-related scales: high Tension and reduced Confidence and Control were associated especially with low Task-Focus and high Emotion-Focus. Cognitive Confidence and MetaWorry were related to tension only. Coping scales were more weakly related to some aspects of task engagement, i.e. energy and concentration. Finally, all three cognitive trait questionnaires related to aspects of DSSQ state worry. Three of the MCQ scales, including Cognitive Self-Consciousness, predicted DSSQ Self-Focus. AnTI scales were most predictive of Task-Related Cognitive Interference, and the CITS-D scales were significantly correlated with Self-Esteem and Task-Related Interference. At the level of factor scores, Metacognition predicted the two Cognitive Interference scales, and Adaptive Coping was related to the three scales indicating low distress, and to high energy. In view of the small magnitudes of most of these relationships, multivariate analyses were not conducted.

Finally, the cognitive trait scales were correlated with the NASA-TLX workload measure, and the CITS-S, measures that indexed the subject's cognitions of the examination situation. Results are shown in Table 7 . The strongest metacognitive predictor was MCQ Uncontrollability and Danger, which related to higher perceived workload, and to use of emotion-focus. Cognitive Confidence and Meta-Worry also correlated with workload. The dispositional coping measures (CITS-D) were appropriately correlated with in-situation coping (CITS-S), although magnitudes of significant correlations were small. All three CITS-S scales were significantly correlated with workload. The Adaptive Coping factor was associated with low workload and high Task-Focus.

\section{DISCUSSION}

Results of this study confirmed that text anxiety traits are substantially related to metacognition, as predicted by cognitive theories of worry. Discussion of results focuses on three issues: cognitive components of test anxiety, comparison of trait and state data and clinical implications.

\section{Metacognition, Coping and Test Anxiety Theory}

Factor analysis of the metacognitive, worry and coping traits distinguished two broad clusters of traits, a general metacognitive factor taking in worry and some of the variance in emotion-focused coping, and a narrower adaptive coping factor. The factors loosely correspond to the Nelson-Narens (1994) distinction between monitoring and control functions. The two factors provided a fairly

Table 7. Correlations of MCQ, AnTI and CITS-D scales and factor scores with NASA-TLX workload and CITS-S scales.

\begin{tabular}{|c|c|c|c|c|}
\hline & \multicolumn{4}{|c|}{ CITS-S scale } \\
\hline & Workload & Task-Focus & Emotion-Focus & Avoidance \\
\hline \multicolumn{5}{|l|}{ Scales } \\
\hline Positive Beliefs & 0.03 & 0.16 & 0.08 & -0.07 \\
\hline Uncontrollability and Danger & $0.23 *$ & -0.02 & $0.22 *$ & 0.11 \\
\hline Cognitive Confidence & $0.27^{*}$ & 0.03 & 0.17 & 0.05 \\
\hline $\mathrm{SPR}^{\mathrm{a}}$ & 0.11 & 0.10 & $0.24 *$ & 0.10 \\
\hline Cognitive Self-Consciousness & -0.02 & 0.05 & 0.05 & 0.12 \\
\hline Social Worry & 0.07 & -0.02 & 0.12 & 0.05 \\
\hline Health Worry & 0.03 & 0.07 & -0.02 & -0.05 \\
\hline Meta-Worry & $0.25 *$ & 0.12 & 0.12 & 0.05 \\
\hline Task-Focus & $-0.24 *$ & $0.28 *$ & -0.20 & -0.17 \\
\hline Emotion-Focus & $0.33^{* *}$ & -0.01 & $0.26^{*}$ & 0.08 \\
\hline Avoidance & $0.26^{*}$ & -0.16 & 0.16 & $0.24^{*}$ \\
\hline \multicolumn{5}{|l|}{ Factor scores } \\
\hline Metacognition & 0.19 & 0.09 & 0.19 & 0.07 \\
\hline Adaptive Coping & $-0.33 * *$ & $0.22 *$ & -0.19 & -0.15 \\
\hline
\end{tabular}

Note. $* p<0.05, * * p<0.01$.

aNegative beliefs about thoughts in general including themes of superstition, punishment and responsibility. 
parsimonious means for describing the overlap between the cognitive traits and test anxiety. Tension was the only RTT scale predicted by individual scales, once the contribution of the two factors was taken into account. Furthermore, the two factors were independently related to the more cognitive aspects of test anxiety, RTT Worry and Test-Irrelevant Thinking. Tension related to general Metacognition, and, independently, to a pattern of coping associated with high emotion-focus and low avoidance. Hence, the data support the predicted associations between test anxiety and 'cognitive traits'. They are also consistent with the view of Matthews et al. (in press-b), that traits tend to relate to multiple processing constructs.

Consistent with the S-REF model (Wells and Matthews, 1994), self-preoccupation in examination settings may partially derive from general metacognitive beliefs, especially beliefs that worrying is uncontrollable and dangerous, as opposed to beliefs that specifically concern the examination situation. The high correlations between MCQ scales and RTT Bodily Symptoms imply that physical test anxiety symptoms may reflect the person's negative metacognitive appraisals as much as actual physiological arousal. The more cognitive aspects of test anxiety (Worry and Test-Irrelevant Thinking) also relate to style of coping with examinations. Use of taskfocused coping may interrupt the cycle of perseverative worry typical of severe anxiety (Matthews and Wells, 1996), and so reduce worry symptoms. However, use of emotion-focus and avoidance, at the expense of task focus, seems to maintain worry about the task and test-irrelevant thoughts.

The factor analysis suggested that the extent to which coping is adaptive partly reflects metacognitions. Cognitive self-consciousness may bias the person towards task-focus in examination settings, whereas beliefs in one's cognitive inefficiency (MCQ Cognitive Confidence) bias emotion-focus and avoidance. As several studies have shown, selfconsciousness does not invariably lead to maladaptive coping (Carver, 1996; Matthews and Wells, 1996). For example, Carver et al. (1983) found that induced self-focus of attention enhanced performance in low-test-anxiety subjects, but impaired performance in test anxious individuals. Such results may be dependent upon metacognitive processes: self-focus may be beneficial when the individuals are sufficiently confident in their own abilities to implement a plan of action. Carver (1996) identifies expectancy of outcome as a key factor moderating the impact of self-focus: negative expectancy and self-focus leads to disengagement from task goals. The MCQ Cognitive Confidence scale may relate to negative expectancy of examination success, biasing the person towards less effective coping. These remarks are tentative, as factor analysis of trait constraints does not assess directly how constructs are interrelated within a dynamic model, such as those of Carver (1996) and Wells and Matthews (1994).

\section{Metacognition and State Expression of Test Anxiety}

The DSSQ data shows that states during the examination were characterized most strongly by elevated scores on the scales contributing to the distress syndrome (see Table 1) as well as by some more specific states such as high task-related cognitive interference, and low intrinsic motivation. The various trait measures were modestly predictive of individual differences in the subjective states experienced. The Adaptive Coping factor was related to lower distress (i.e. lower tension etc.) and to greater energy, whereas the Metacognition factor predicted both Cognitive Interference dimensions. Three of the MCQ scales predicted state SelfFocus, although the Metacognition factor did not. The trait measures also correlated with subject's cognitions of the examination: workload appraisal and choice of coping strategy. For example, the Adaptive Coping factor predicted lower workload and more use of task-focus, and two of the MCQ scales related to use of emotion-focus. However, metacognition and coping correlates of traits and states did not correlate closely. For example, Adaptive Coping was most predictive of cognitive aspects of test anxiety at the trait level (e.g. RTT Worry), but most predictive of emotional aspects of the state level (e.g. DSSQ Tension). The Metacognition factor was generally less predictive at the state than the trait level, although it did predict both trait and state cognitive interference. Individuals with high scores on the Metacognition factor report on the RTT that examinations make them feel tense, but Metacognition did not predict DSSQ tension (although MCQ Cognitive Confidence and AnTI Meta-Worry were predictive of tension).

There are three possible reasons for discrepancies between trait and state correlates of test anxiety. First, both cognitive personality theory (Matthews et al., in press-b) and test anxiety theory (Sarason et al., 1986) emphasize the interaction of anxietyrelated traits with situational factors in generating state anxiety symptoms. Various situational factors related to the classroom and test environment seem 
to moderate relationships between test anxiety and performance (see Zeidner, 1998). It is difficult to assess situational factors post hoc, but perhaps the particular examination used in the present study was not representative of the past examination experiences that guided subjects' responses on the RTT. The RTT was only weakly predictive of postexamination state, with only the Worry scale relating substantially to DSSQ scores and cognitions of the task. A previous study using the DSSQ (Matthews et al., 1996) found considerably stronger correlations between RTT scales and state scales related to distress and worry following an end-ofyear psychology examination.

Second, scores on the RTT may be influenced by memory biases. In particular, 'high-metacognition' subjects may be prone selectively to recall particularly unpleasant examination experiences. Plausibly, metacognitive traits encourage rumination on unpleasant experiences, leading to more accessible memories subsequently. Third, temporal variation in stress reactions during examinations may require attention (Zeidner, 1991). The DSSQ requires subjects to rate the feelings during the task just completed. However, a substantial proportion of RTT items refer to feelings before the test; e.g. 6/10 items on the Tension scale. Subjects high on the Metacognition factor may experience high anticipatory tension, which is alleviated when the examination begins and attention is, at least partially, diverted from the self to the task. Future research could usefully assess state metacognition within the examination situation, perhaps at various time-points.

\section{Clinical Implications}

The data suggest that modification of metacognition and maladaptive coping may provide the therapist with several distinct techniques for treating test anxiety. First, therapy may be directed towards altering the self-knowledge that generates excessive metacognitive processing during test performance (and other situations). Second, the therapist may work towards decoupling metacognition in the test situation from the person's general metacognitive style, so that attention remains predominantly task focused. Third, the problem may stem from inadequate coping skills, requiring training in the use of task-focus in place of emotion-focus and avoidance.

The first therapeutic avenue, restructuring selfknowledge, is the most radical, in that it seeks to modify the cognitive bases for personality. The associations between test anxiety and general style of metacognition imply that test anxiety may partially reflect cognitive aspects of personality that are more pervasive than a specific problem with evaluation situations. It is plausible that the techniques of cognitive-behaviour therapy for generalized anxiety and worry (Wells, 1997) can be applied straightforwardly to test anxiety. Wells (1997) advocates that interventions should focus primarily on challenging metaworry, rather than teaching anxiety management. Treatment of test anxiety might then include some of the following components. Verbal reattribution techniques might include challenging metacognitive beliefs that worry is useful for problem-solving or that worries experienced during examinations are uncontrollable. Behavioural experiments might be contrived during which the person answers examination questions under conditions controlled by the therapist. For example, the person might be permitted to worry prior to or during the 'examination'. In this way beliefs about the uncontrollability of worry and its perceived advantages can be challenged. Such techniques are perhaps most suitable for severe or incapacitating test anxiety, or cases in which test anxiety is one expression of a more general anxiety problem.

Second, interventions may be directed towards specific coping skills rather than towards metacognition. Training of coping skills to reduce cognitive interference has mainly been explored in the context of competitive sports anxiety (see Nideffer, 1993; Smith, 1996). However, some of the techniques listed by Smith (1996) might also be applied to examination anxiety. Effective skill instruction in the test anxiety context refers to training in examination technique: time management, planning of essay answers and so forth (see Mueller, 1992). Explicit training may boost expectancy of success, and so reduce worry, as well as improving objective skills. It may also be important to teach effective study skills, which are lacking in some test anxious individuals (Mueller, 1992). Standard stress management interventions such as relaxation techniques are demonstrably effective in reducing cognitive interference in the sports context (see Smith, 1996). Other techniques such as mental rehearsal, breathing techniques and ritualized pre-performance routines which focus attention on the task may also be applicable to the test situation. However, the present data suggest that test anxiety has a metacognitive aspect which is distinct from maladaptive coping. The RTT data here imply that training coping skills would be more effective for 
test anxious individuals not prone to metaworry, and more effective for cognitive than for emotionalsomatic aspects of test anxiety. Training coping may also be more effective for relatively moderate levels of test anxiety. In more severe cases it has various disadvantages such as failure to address underlying problems at the self-knowledge level (Matthews and Wells, 1996). Instruction in coping may, of course, be a useful adjunct to therapies directed towards restructuring self-knowledge.

A third approach to therapy is to reduce the situational expression of test anxiety as cognitive interference and worry states, by training the specific coping skill of attentional control. Experimental studies of techniques for reducing cognitive interference have focused on this type of treatment (e.g. Sarason et al., 1990). For example, Sarason (1984) showed that attention-directing and reassurance manipulations improved performance in subjects with high scores on the RTT Worry scale. Instructions to direct attention to the task were most successful overall, because low-worry subjects tended to perform poorly with reassuring instructions. We might suspect that reassurance tends to reduce worry-related metacognitions, but also reduces task-focused/adaptive coping. Other studies have confirmed that training subjects to focus on the task is an effective treatment for test anxious students, although decreased anxiety does not always lead to improved test performance (see Zeidner, 1998). Attention training techniques (Wells, 1990; Wells et al., 1997) developed for clinical anxiety patients might also be used to train executive control during examinations systematically. Such techniques have been effective in treatment of a variety of anxiety conditions including panic and social phobia. In contrast to the relatively simple instructional treatments sometimes used to control test anxiety, Wells' attention training may help the patient to restructure maladaptive self-knowledge, as well as facilitating situationspecific attentional control. Hence, attention training of this kind might be effective when test anxiety is associated with both metacognitive and coping dysfunction.

\section{CONCLUSIONS}

The study described here suggests that test anxiety traits are closely related to a 'general metacognition' factor defined most strongly by beliefs that thoughts are uncontrollable, metaworry and use of emotion-focused coping. In addition, a separate 'adaptive coping' factor contributes independently to cognitive expressions of test anxiety. Both factors were also implicated in test anxiety states during an actual examination, although further work is needed to resolve some discrepancies between state and trait level data. Various options for therapy for test anxiety were set out in the light of these findings. Because test anxiety seems to relate to multiple aspects of cognition, it is important to examine the sources of test anxiety in the individual in some detail. Different treatment options may be preferred depending on whether anxiety is primarily metacognitive or coping-based.

\section{ACKNOWLEDGEMENT}

This research was supported by a Medical Research Council ROPA Award to the first author. The DSSQ and CITS are available to qualified psychologists from the first author.

\section{REFERENCES}

Cartwright-Hatton, S. and Wells, A. (1997). Beliefs about worry and intrusions: the Meta-Cognitions Questionnaire and its correlates. Journal of Anxiety Disorders, 11, 279-296.

Carver, C. S. (1996). Cognitive interference and the structure of behavior. In: I. G. Sarason, G. R. Pierce and B. R. Sarason (Eds), Cognitive Interference: Theories, Methods, and Findings. Mahwah, NJ: Erlbaum, pp. 25-45.

Carver, C. S., Peterson, L. M., Follansbee, D. J. and Scheier, M. F. (1983). Effects of self-directed attention and resistance among persons high and low in testanxiety. Cognitive Therapy and Research, 7, 333-354.

Costa, P. T. Jr, Somerfield, M. R. and McCrae, R. R. (1996). Personality and coping: a reconceptualization. In: $\mathrm{M}$. Zeidner and N. S. Endler (Eds), Handbook of Coping: Theory, Research, Applications. New York: Wiley, pp. 44-61.

Endler, N. S. and Parker, J. D. A. (1990). Multi dimensional assessment of coping: a critical review. Journal of Personality and Social Psychology, 58, 844-854.

Geen, R. G. (1987). Test anxiety and behavioral avoidance. Journal of Research in Personality, 21, 481-488.

Glass, C. R., Arnkoff, D. B., Wood, H., Meyerhoff, J. L., Smith, H. R., Oleshansky, M. A. and Hedges, S. M. (1995). Cognition, anxiety, and performance on a career-related oral examination. Journal of Counseling Psychology, 42, 47-54.

Hart, S. G. and Staveland, L. E. (1988). Development of a multidimensional workload rating scale: results of empirical and theoretical research. In: P. A. Hancock and N. Meshkati (Eds), Human Mental Workload. Amsterdam: Elsevier. 
Hendy, K. C., Hamilton, K. M. and Landy, L. N. (1993). Measuring subjective workload: when is one scale better than many? Human Factors, 35, 579-601.

Horn, J. L. (1965). A rationale and test for the number of factors in factor analysis. Psychometrika, 30, 179-185.

Matthews, G. and Campbell, S. E. (1998). Task-induced stress and individual differences in coping. Proceedings of the 42nd Annual Meeting of the Human Factors and Ergonomics Society, pp. 821-825.

Matthews, G., Campbell, S. E., Joyner, L., Huggins, J., Falconer, S. and Gilliland, K. (1996). The Dundee Stress State Questionnaire: an Initial Report. Unpublished technical report, University of Dundee, .

Matthews, G. and Deary, I. (1998). Personality Traits. Cambridge: Cambridge University Press.

Matthews, G., Joyner, L., Gilliland, K., Campbell, S. E., Falconer, S. and Huggins, J. (in press-a). Validation of a comprehensive stress state questionnaire: towards a state 'Big Three'? In: I. J. Mervielde, F. Deary, F. De Fruyt and F. Ostendorf (Eds), Personality Psychology in Europe (Vol. 7). Tilburg: Tilburg University Press.

Matthews, G., Saklofske, D. H., Costa, P. T. Jr, Deary, I. J. and Zeidner, M. (1998). Dimensional models of personality: a framework for systematic clinical assessment. European Journal of Personality Assessment, 14, 35-48.

Matthews, G., Schwean, V. L., Campbell, S. E., Saklofske, D. H. and Mohamed, A. A. R. (in press-b). Personality, self-regulation and adaptation: a cognitive-social framework. In: M. Boekarts, P. R. Pintrich and M. Zeidner (Eds), Handbook of Self-Regulation. New York: Academic.

Matthews, G. and Wells, A. (1996). Attentional processes, coping strategies and clinical intervention. In: $\mathrm{M}$. Zeidner and N. S. Endler (Eds), Handbook of Coping: Theory, Research, Applications. New York: Wiley, pp. 573-601.

Mueller, J. H. (1992). Anxiety and performance. In: A. P. Smith and D. M. Jones (Eds), Handbook of Human Performance, Vol. 3: State and Trait. London: Academic, pp. 127-160.

Nelson, T. O. and Narens, L. (1994). Why investigate metacognition? In: J. Melcalfe and A. P. Shimamura (Eds), Metacognition: Knowing about Knowing. Cambridge, MA: MIT Press.
Nideffer, R. M. (1993). Concentration and attention control training. In: J. M. Williams (Ed.), Applied Sport Psychology: Personal Growth to Peak Performance (2nd edn). Palo Alto, CA: Mayfield, pp. 243-261.

Sarason, I. G. (1984). Test anxiety, stress, and cognitive interference: reactions to tests. Journal of Personality and Social Psychology, 46, 929-938.

Sarason, I. G., Sarason, B. R., Keefe, D. E., Hayes, B. E. and Shearin, E. N. (1986). Cognitive interference: situational determinants and traitlike characteristics. Journal of Personality and Social Psychology, 51, 215-226.

Sarason, I. G., Sarason, B. R. and Pierce, G. R. (1990). Anxiety, cognitive interference, and performance. Journal of Social Behavior and Personality, 5, 1-18.

Smith, R. E. (1996). Performance anxiety, cognitive interference, and concentration enhancement strategies in sports. In: I. G. Sarason, G. R. Pierce and B. R. Sarason (Eds), Cognitive Interference: Theories, Methods, and Findings. Mahwah, NJ: Erlbaum, pp. 261-283.

Wells, A. (1990). Panic disorder in association with relaxation-induced anxiety: an attentional training approach to treatment. Behavior Therapy, 21, 273-280.

Wells, A. (1994). A multi-dimensional measure of worry: development and preliminary validation of the Anxious Thoughts Inventory. Anxiety, Stress and Coping, 6, 289-299.

Wells, A. (1997). Cognitive Therapy of Anxiety Disorders: A Practice Manual and Conceptual Guide. Chichester: Wiley.

Wells, A. and Matthews, G. (1994). Attention and Emotion: A Clinical Perspective. Hove: Erlbaum.

Wells, A. and Matthews, G. (1996). Modelling cognition in emotional disorder: the S-REF model. Behaviour Research and Therapy, 34, 881-888.

Wells, A., White, J. and Carter, K. (1997). Attention training: effects on anxiety and beliefs in panic and social phobia. Clinical Psychology and Psychotherapy, 4, 226-232.

Zeidner, M. (1991). Test anxiety and aptitude test performance in an actual college admissions testing situation: temporal considerations. Personality and Individual Differences, 12, 101-109.

Zeidner, M. (1998). Test Anxiety: The State of the Art. New York: Plenum.

Zwick, W. and Velicer, W. (1986). Comparison of five rules for determining the number of components to retain. Psychological Bulletin, 99, 432-442. 